\title{
Confirmada la presencia de Crucianella patula L. (Rubiaceae) en Andalucía occidental (España)
}

\author{
Gloria Martínez-Sagarra* \& Juan Antonio Devesa \\ Departamento de Botánica, Ecología y Fisiología Vegetal, Facultad de Ciencias, Universidad de Córdoba. Edificio José \\ Celestino Mutis, Campus de Rabanales, Universidad de Córdoba, 14071 Córdoba (España).
}

\section{Correspondencia}

G. Martínez-Sagarra

e-mail: bv2masag@uco.es

Recibido: 25 junio 2020

Aceptado: 10 julio 2020

Publicado on-line: 27 julio 2020

Editado por: Marta Recio Criado

\section{Resumen}

Se da a conocer por primera vez la presencia de Crucianella patula L. en la provincia de Córdoba, y esta nueva cita respalda su inclusión en la flora de Andalucía Occidental.

Palabras clave: Flora vascular, Córdoba, Corología, Crucianella.

\begin{abstract}
Confirmed presence of Crucianella patula L. (Rubiaceae) in western Andalusia (Spain)

The presence of Crucianella patula L. in the province of Cordoba is reported for the first time, and this new record supports its inclusion in the flora of western
\end{abstract} Andalusia.

Keywords: Vascular flora, Cordoba, Chorology, Crucianella.
Al revisar materiales no identificados del herbario de la Facultad de Ciencias de la Universidad de Córdoba (COFC), se han detectado dos poblaciones de Crucianella patula L. en la provincia de Córdoba, para cuya flora la especie constituye una novedad. Además, el hallazgo confirma el taxón para la flora de Andalucía Occidental, donde su presencia había sido cuestionada.

Crucianella patula L., Demonstr. PI.: 4 (1753)

CÓRDOBA. Baena, Gastaceite, olivar, 30SUG8572, 17-V-1988, sin recolector (COFC 66060); Luque, ctra. N-321, km 113, 30SUG9758, 3-VI-1980, M.L. Díaz \& J. Muñoz (COFC 70794).

Especie descrita de España y que se distribuye por el centro, este y sur de la Península Ibérica, Islas Baleares, y noroeste de África (Devesa, 2007). Las localidades indicadas son las únicas de la especie conocidas en la provincia de Córdoba, y su existencia constituye una novedad para su flora. Además, el hallazgo confirma también el taxón para flora vascular de Andalucía Occidental, en la que no obstante había sido incluida para la provincia de Cádiz (Devesa, 1987) sobre la base de una cita de Lange [1868;
"Baet. (Cadiz, CABR. in hb. AGARDH!)"], nunca confirmada, y que más tarde recogió Pérez Lara [1889; "Cádiz (Cabrera, in herb. Agardh ex Lange)"]. Precisamente por ello, en el tratamiento del género para Flora iberica, se puso en duda la existencia de la especie en dicha provincia (cf. Devesa, 2007), pero con esta cita queda inequívocamente confirmada su presencia al menos en las Sierras Subbéticas cordobesas.

\section{Bibliografía}

Devesa, J. A. (1987). Crucianella. In: B. Valdés et al. (Eds.) Flora Vascular de Andalucía Occidental, 2 (pp. 575-577). Barcelona: Ketres Editora S. A.

Devesa, J. A. (2007). Crucianella. In: S. Castroviejo et al. (Eds.) Flora iberica XV (pp. 30-36). Madrid. Departamento de publicaciones del CSIC.

Lange, J. (1868). Crucianella. In: H.M. Willkomm \& J.M.C. Lange (Eds.) Prodromus Florae hispanicae 2, 305-306. Stuttgart.

Pérez Lara, J.M. (1889). Florula Gaditana... Pars Tertia. Anales de la Sociedad Española de Historia Natural 18: 35-143. 